\title{
Thoracic Epidural Plus Continuous Brachial Plexus Analgesia for Pediatric Reconstructive Surgery: A Case Report
}

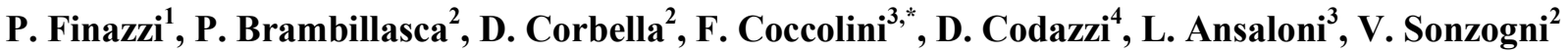 \\ ${ }^{1}$ Brescia University, Anesthesiology dept., Brescia, Italy \\ ${ }^{2}$ Anesthesiology dept., General Surgery dept. Papa Giovanni XXIII hospital, Bergamo, Italy \\ ${ }^{3}$ General Surgerydept., Papa Giovanni XXIII Hospital, Bergamo, Italy \\ ${ }^{4}$ Plastic Surgery dept., Papa Giovanni XXIII Hospital, P.zza OMS 1, 24127 Bergamo, Italy \\ *Corresponding author: federico.coccolini@gmail.com
}

Copyright (C) 2013 Horizon Research publishing all rights reserved.

\begin{abstract}
We present a case of an 11-years old child who underwent several plastic procedures for massive skin necrosis of the right forearm. Pain control was achieved combining a thoracic epidural and a continuous brachial plexus block. The patient was successfully weaned from chronic opiates. Possible advantages (i.e. improved graft perfusion, speeded opiates weaning) and disadvantages (i.e. local anesthetic toxicity) of this combined approach are briefly discussed.
\end{abstract}

Keywords Thoracic Epidural, Continuous Brachial Plexus Block, Pediatric Loco-Regional Anesthesia, Local Anesthetic Toxicity, Opiates Weaning

\section{Introduction}

Severe and uncontrolled pain is related to perioperative morbidity and mortality, prolonged hospital stay and patient dissatisfaction.

During the last two decades the loco-regional anesthesia became part of the multimodal approach to pain control even in the pediatric population. Its implementation showed a superior pain control and fewer side effects than the use of systemic therapies such as parenteral opioids [1]. Beside these, regional anesthesia-analgesia showed specific features as reduced hormonal response, perioperative blood loss, gastrointestinal and host-defense dysfunction, as recently reviewed [1].

Anyway, a systemic administration of analgesics can be mandatory when a surgical procedure involves multiple body-district that can not be covered by a single loco-regional technique. To overcome the problem more than a single regional technique can be used[2]. As multiple and concomitant infusions of local anesthetics are performed, the patient is at increased risk to reach the toxic dose [3].
We face more and more frequently the problem of treating acute post-operative pain on a chronic pain substrate, as it is in this report[4]. The two main challenges of this scenario are how to treat a patient with a chronic opiates use and how cover the area of hypersensitization around the wounds. Moreover, we need to consider that the acute post-operative pain is longer than usual so the chosen pain control technique needs to be suitable for a longer period too.

\section{Materials and Methods}

An 11 years-old, $28 \mathrm{~kg}$, boy was transferred, from a refugee camp in Congo, to Italy thanks to a Humanitarian International Program. Five years ago, the patient developed a severe Volkmann's ischemic contracture [5] (compartment syndrome involving the muscles of the forearm) associated with skin chronic ulcers (from the proximal third of the right arm to the wrist) due to the toxic effect of snakebite. The fourth finger of the right hand was probably amputated because of incoming necrosis. Cultural skin sampling showed infection/contamination by multiple bacterias secondary to poor hygienic conditions and lack of medical support.

After his admission in Plastic Surgery Department he underwent multiple surgical debridement, to reduce microbiological charge, under sedation and/or general anesthesia. Antibiotic therapy was modulated on specific antibiogramme.

Intravenous opiates were used for pain control during the procedures. The patient had nausea and vomiting, and irritability as a consequence of their use.

After negativization of microbiological wound culture (day 28), plastic surgeons placed three tissue expanders (Mentor, 201 Mentor Drive, Santa Barbara, CA 93111) under sub-fascial pockets in right lumbar and iliac regions.

For seven weeks the expanders were systematically and rapidly filled to their maximal volume in operating room. On 
day 77, plastic surgeons removed the skin expanders and designed two thoraco-epigastric skin/fascia flaps to cover arm and forearm. During the same procedure multiple Kirschner's wires were placed in elbow, wrist and fingers in functional position.

We used for induction intravenouspropofol $(2 \mathrm{mg} / \mathrm{kg})$, fentanyl $(2 \mathrm{mcg} / \mathrm{kg})$, and Sevoflurane $2.5 \%$ for maintenance. In the immediate post-operative, in PACU, with patient still under GA, an epidural catheter, T7-T8, was placed to cover the wound of the abdominal pocket (Ropivacaine $0,1 \%+$ Sufentanil $1 \mathrm{mcg} / \mathrm{mL}$ at $4 \mathrm{~mL} / \mathrm{h}$ ). A brachial plexus catheter was also placed with an ultrasound-guided supraclavicular technique $[6,7]$ (Ropivacaine $0,2 \%, 4 \mathrm{~mL} / \mathrm{h}$ ). The recommended hourly dose for epidural infusion of Ropivacaine in children older than 6 months $(0,4 \mathrm{mg} / \mathrm{kg} / \mathrm{h})$ was considered as maximum safe dose[3, 8]. To overcome this limit we used a diluted concentration $(0,1 \%)$ in continuous infusion through the epidural route. Intravenous acetaminophen was set as rescue. Even though the infusion rate of Ropivacaine was slightly upon the safety limit $(0.42$ $\mathrm{mg} / \mathrm{kg} / \mathrm{h}$ for a prolonged period of time) we planned not to sample Ropivacaine plasma concentration level and to rely only upon clinical monitoring of neurological signs of local anesthetic toxicity. We felt confident with this approach as the threshold limit was posed for Bupivacaine continuous infusion while we used Ropivacaine that has a better toxicity profile and less tendency to accumulation $[3,8]$.

The patient was sent to PICU, extubated in three hours and discharged in post-operative day 3 .

The pedicles of the flaps were divided on day 105 (28 days after flaps coverage). The donor defect and those areas uncovered by the flaps were covered by INTEGRA ${ }^{\circledR}$ Dermal Regeneration Template (SIAD Healthcare S.p.a. Milano, Italy). We repeated the same anesthesia/analgesia scheme of the previous procedure with the placement of a thoracic epidural catheter and a brachial plexus catheter and iv acetaminophen as rescue.

At day 175, plastic surgeons removed the silicon sheet on INTEGRA ${ }^{\circledR}$ and covered it with skin grafts.

\section{Results}

The perioperative pain control flap coverage was excellent (Visual Analog Score (VAS) 0-2) until post-operative day 6 when he showed pain (VAS 4-5) in the ulnar innervation area. Intravenous acetaminophen $400 \mathrm{mg}$ four times a day was enough to control pain. The epidural catheter was removed on day 5 , the peri-neural one on day 11 . US of supraclavear area detected no signs of infection.

After the division of flaps' pedicles, we reported a similar pain scheme, with a VAS peak on day 2 (4-2), due to physical rehabilitation, and similarly treated with acetaminophen. After this procedure we removed the epidural catheter on day 7 and the supraclavear one on day 11 (VAS score 0-2). Similarly, no signs of infections were detected at US scan.

\section{Conclusions}

The use of a multimodal analgesic regimen, with loco-regional continuous analgesia resulted in a very rapid discharge from PICU. It prevented from the use of around-the-clock intravenous analgesic. Moreover, the patient was successfully and completely weaned from opioids, used in the early post-operative stage, with their gastrointestinal and behavioral side effects.

Loco-regional continuous analgesia can also guarantee sympathetic blockade that is useful in providing constant rise in regional diastolic pressure. It may enhance vascularization of flaps and their inset in recipient site[9].

Despite the presence of two simultaneous infusions of local anesthetic,we had no local anesthetics toxicity and achieved a good pain control in a large body-district area.

\section{Conflict of Interest}

All authors declare to have no conflict of interest

\section{REFERENCES}

[1] Wolf AR. Effects of regional analgesia on stress responses to pediatric surgery. Paediatr Anaesth. 2012 Jan;22(1):19-24.

[2] Dadure C, Capdevila X. Peripheral catheter techniques. Paediatr Anaesth. 2012 Jan;22(1):93-101.

[3] Berde CB. Toxicity of local anesthetics in infants and children. J. Pediatr. 1993 May;122(5 Pt 2):S14-20.

[4] Geary T, Negus A, Anderson BJ, Zernikow B. Perioperative management of the child on long-term opioids. Paediatr Anaesth. 2012 Mar;22(3):189-202.

[5] Prasarn ML, Ouellette EA. Acute compartment syndrome of the upper extremity. J Am Acad Orthop Surg. 2011 Jan;19(1):49-58.

[6] Marhofer P, Willschke H, Kettner SC. Ultrasound-guided upper extremity blocks - tips and tricks to improve the clinical practice. Paediatr Anaesth. 2012 Jan;22(1):65-71.

[7] De José María B, Banús E, Navarro Egea M, Serrano S, Perelló M, Mabrok M. Ultrasound-guided supraclavicular vs infraclavicular brachial plexus blocks in children. Paediatr Anaesth. 2008 Sep;18(9):838-44.

[8] Bösenberg AT, Thomas J, Lopez T, Huledal G, Jeppsson L, Larsson LE. Plasma concentrations of ropivacaine following a single-shot caudal block of 1,2 or $3 \mathrm{mg} / \mathrm{kg}$ in children. Acta Anaesthesiol Scand. 2001 Nov;45(10):1276-80.

[9] Landsverk SA, Kvandal P, Kjelstrup T, Benko U, Bernjak A, Stefanovska A, et al. Human skin microcirculation after brachial plexus block evaluated by wavelet transform of the laser Doppler flowmetry signal. Anesthesiology. 2006 Sep;105(3):478-84. 